\title{
Subcutaneous granuloma annulare: a case report
}

\author{
Granuloma anular subcutáneo: reporte de un caso
}

\author{
Francisco Reyes-Baraona ${ }^{a}$, Paula Hasbún ${ }^{b}$, Sergio Gonzálezc, María Soledad Zegpi ${ }^{a}$
}

aDepartment of Dermatology, Faculty of Medicine, Pontificia Universidad Católica of Chile

bPrimary Specialty Center San Lázaro, Santiago of Chile

'Department of Pathology, Faculty of Medicine, Pontificia Universidad Católica of Chile

Fecha recibido: 12-11-16; Fecha aceptado: 17-12-2016

\begin{abstract}
Introduction: Granuloma annulare is a benign and self-limited cutaneous disease. Subcutaneous granuloma annulare is an uncommon variant seen almost exclusively in young children, characterized by firm nodules appearing more frequently in lower extremities, buttocks, hands and scalp. Objective: To report a case of subcutaneous granuloma annulare and review its differential diagnoses and treatments. Clinical case: A 4 year-old male patient. Since 2 and a half years of age he presented asymptomatic firm subcutaneous nodules on the back of the left middle finger and later on the back of the left hand, right big toe, left frontal area and scalp. Excisional biopsy of some lesions from the scalp was consistent with subcutaneous granuloma annulare. Clobetasol 0,05\% cream twice a day for 1 month was prescribed without response. Conclusions: Subcutaneous granuloma annulare must be considered within the differential diagnosis of subcutaneous nodules in children. In most of the cases a biopsy and histopathology of the lesions will be required to confirm the diagnosis. Due to its tendency to spontaneous resolution, treatment often is not necessary and periodic evaluation is recommended.
\end{abstract}

\section{Keywords:}

Granuloma annulare, subcutaneous granuloma annulare, subcutaneous nodules

\section{Introduction}

The granuloma annulare (GA) is an inflammatory granulomatous dermatitis, benign and self-limited. It has been reported variants with different clinical characteristics, the five most frequent are: localized or classic, generalized, subcutaneous, puncture and in patches ${ }^{1}$. The subcutaneous granuloma annulare is an uncommon variant of unknown prevalence. Most frequently found in younger children, between 2 and 5 years old, predominantly in females ${ }^{2}$, clinically characterized by nodules of 0.24 to 1.3 in $(6 \mathrm{~mm}$ to $3.5 \mathrm{~cm})$ in diameter, of firm consistency, often asymptomatic, located in the deep dermis and hypodermis. Lower limbs are the most affected areas, especially the pretibial area, followed by buttstocks, hands, and scalp. ${ }^{3}$ 
There may be concomitantly lesions in the classic variant in up to $25 \%$ of cases ${ }^{4}$, characterized by skincolored papules, violet or erythematous, that converge in a semi or complete circle, forming an annular lesion.

The objective of this study is to report a subcutaneous granuloma annulare case due to its low frequency, analyzing its differential diagnosis and current therapeutic options.

\section{clinical case}

A 4-year-old male patient, with history of bilateral toe syndactyly and delayed psychomotor development. Since he was two and half years old, he had a single lesion, asymptomatic, on the back of the proximal interphalangeal joint (PIJ) of the left middle finger. A year later, multiple similar lesions appeared close to the first lesion and also on the back of the third left metacarpal phalangeal joint (MPJ), on the right big toe, on the left frontal zone, and on the scalp.

The physical examination showed 6 indurated, skin-colored, subcutaneous nodules on the left frontal region and 2 on the left parietal region, skin-colored papules grouped on the back of the third left metacarpal phalangeal joint (MPJ) (figure 1), skin-

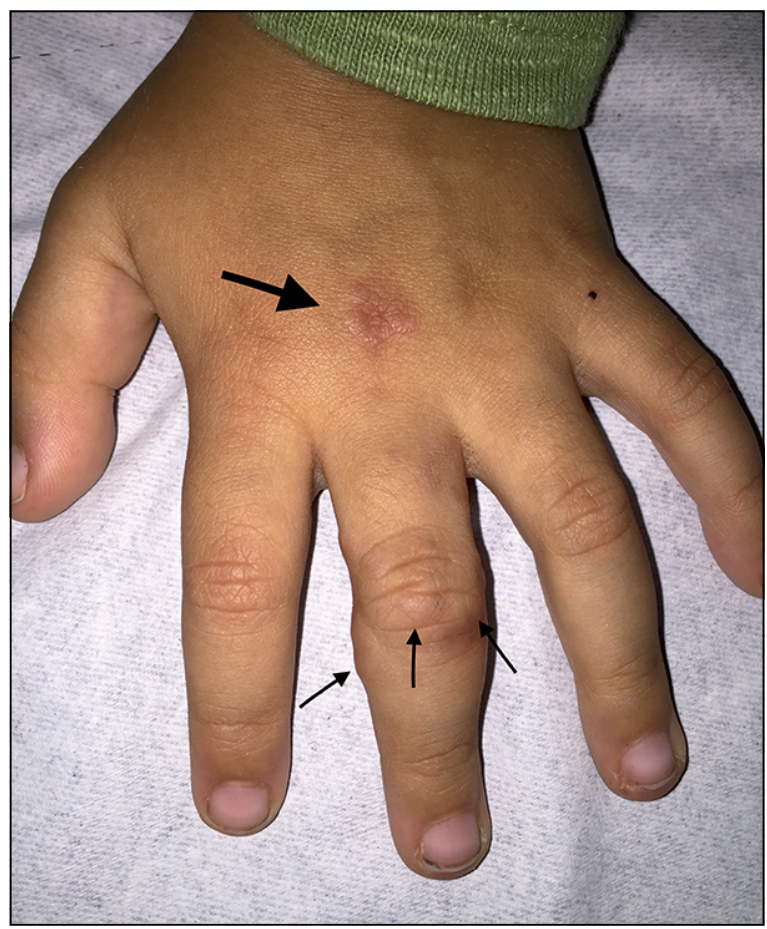

Figure 1. Skin colored papules and nodules on the back and around the 3rd joint left IPJ (thin arrows). Skin colored papules with annular configuration grouped on the back of the 3rd left MCP joint (thick arrows). colored papules and nodules on the back and around the third left interphalangeal proximal joint (PIJ) (figure 1), and 1 skin-colored nodule on the back of the right big toe.

The ultrasound in soft tissue lesions showed hypoechogenic formations of different sizes, with no flow alteration to Doppler that could be related to fibromatous involvement.

The excisional biopsy of some scalp lesions showed dermis and hypodermis with nodular swelling with granulomas of palisaded histiocytes in the necrobiotic center and mucinosis (figure 2 and 3 ), which is compatible with subcutaneous granuloma annulare.

The indicated treatment was clobetasol $0.05 \%$ cream twice a day on the lesions for a month, then there were no changes in the lesions.
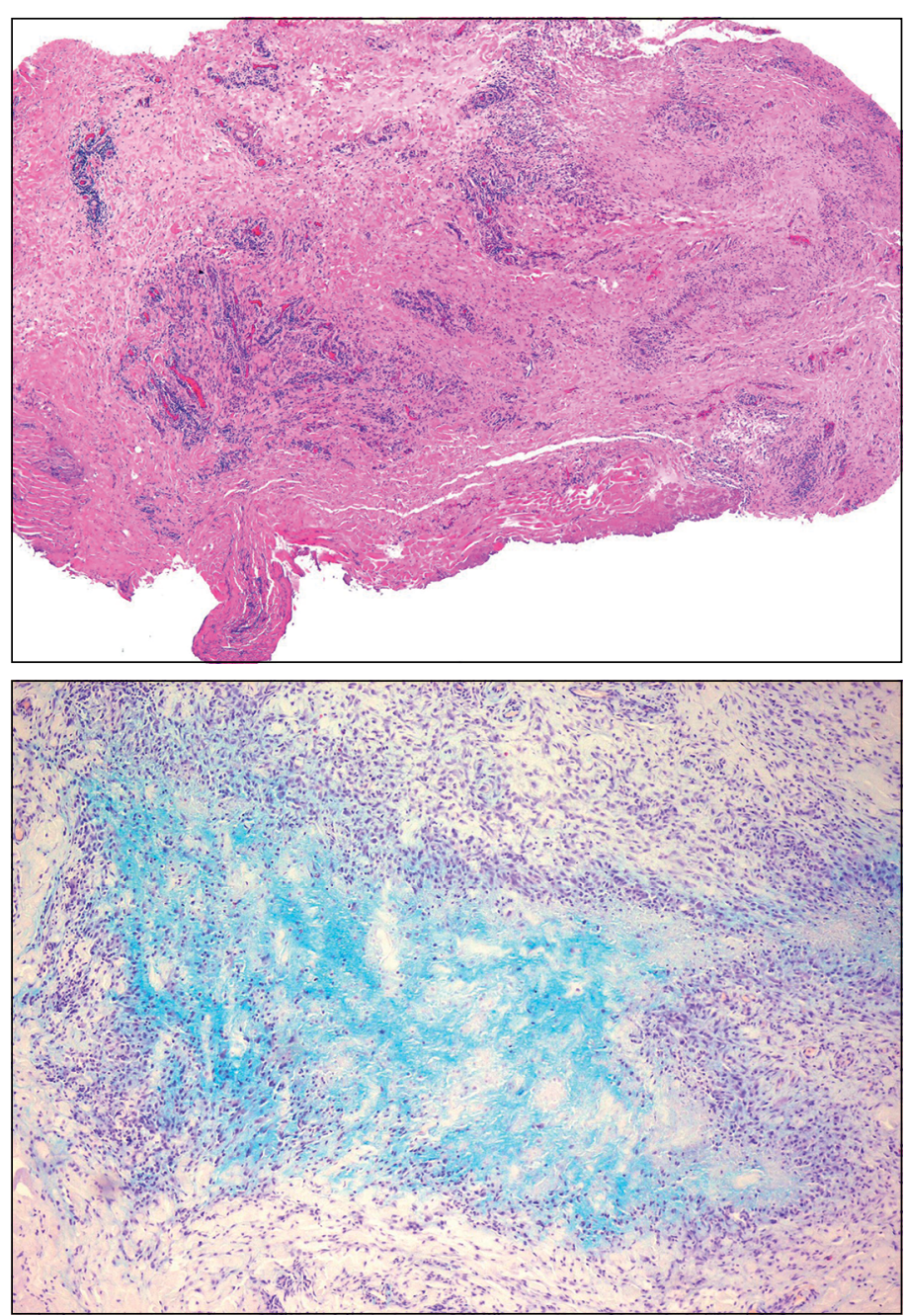

Figure $\mathbf{2}$ and $\mathbf{3}$. Nodules of dense connective tissue located in the deep dermis associated to palisade granulomas with necrobiotic center and abundant mucin deposition. 


\section{Discussion}

The cause of subcutaneous granuloma annulare remains unknown. Some mechanisms have been proposed such as physical trauma, infections (streptococci, tuberculosis, Epstein-Barr virus, and herpes zoster), immunizations, bug bites, diabetes mellitus, and alterations in the cell-mediated immune responses. ${ }^{5}$

The histopathology shows an infiltrate in the deep dermis and/or hypodermis of granulomas predominantly formed by palisaded histiocytes around a central region of degenerating collagen fibers (necrobiosis) and abundant mucin, best seen under alcian blue staining that provides a blue coloration under the microscope. $^{6}$

This is a characteristic of 'blue granulomas', which is also observed in rheumatoid nodules and epithelioid sarcoma.

The subcutaneous granuloma annulare is a pathology that shares clinical and histopathological similarities with other disorders. In the differential diagnosis, it is possible to find rheumatoid nodules, epithelioid sarcoma, tendinous xanthoma, subcutaneous sarcoidosis, erythema nodosum, dermoid cysts, and deep granulomatous infections, among others. ${ }^{1}$

The epithelioid sarcoma is a malignant tumor of the soft tissue, which initially appears as a single, firm, and painless nodular lesion, mainly located in forearms, hands, and fingers. Due to its harmless initial appearance, It can take months or years before the patient seeks medical attention, thus it is common to find an advanced disease or metastasis at the time of the diagnosis. ${ }^{7}$ The epithelioid sarcoma is more frequent in young adults, unlike the subcutaneous granuloma annulare which is more common in children, however, it is a differential diagnosis that must be dismissed in every nodular subcutaneous lesions.

The subcutaneous nodules of rheumatoid arthritis are firm, painless and mobile. Their sizes range from 0.08 to 2 in $(2 \mathrm{~mm}$ to $5 \mathrm{~cm})$ and they usually appear on the extensor surfaces of the periarticular zones and areas subject to pressure or trauma. Patients with rheumatoid nodules tend to develop a more severe rheumatoid arthritis and it is common to find positive anticyclic citrullinated peptide antibodies. ${ }^{8}$

In the subcutaneous sarcoidosis, the nodules are skin-colored, erythematous or hyperpigmented, asymptomatic or slightly painful, and they are mainly located in the upper limbs. Its higher incidence happens in the fourth decade of life, and it is more frequent in female patients. ${ }^{9}$

The dermoid cysts differ from the subcutaneous granuloma annulare because they are congenital lesions, usually distributed in throughout the facial embryologic fusion planes or within the neutral axis.
The most frequent localization is the bregma, around of anterior fontanelle, the forehead lateral-superior region, the lateral region of the upper eyelid, and the submental region. The nodules are subcutaneous, their size ranges from 0.3 in to 1.5 in ( 1 to $4 \mathrm{~cm}$ ), painless, and usually appear individually. It is more frequent to find a connection with the central nervous system when they are located in the midline. ${ }^{10}$

Due to the similarity of the subcutaneous granuloma annulare with the mentioned pathologies, most of the times a biopsy and a histopathologic study of the lesions are required in order to confirm the diagnosis.

The subcutaneous granuloma annulare tends to disappear spontaneously, thus it is suggested not to treat it but control it periodically. ${ }^{1}$ Within the therapeutic alternatives, the high-potency topical corticosteroids such as clobetasol $0.05 \%$ twice a day or the intralesional corticosteroids are the first option, although with variable responses. Other less studied topical therapeutic alternatives include cryotherapy, laser therapy, electrocoagulation, PUVASOL, and topical imiquimod. ${ }^{5}$

\section{Conclusion}

This case of subcutaneous granuloma annulare was reported since it is a rare dermatologic pathology that mainly occurs in children. In the clinical practice, the pediatrician will treat many patients looking for medical advice due to the presence of asymptomatic subcutaneous nodules that in the physical examination seems to be unspecific. He must consider within the differential diagnosis the subcutaneous granuloma annulare. Although it is a benign, self-limited disease, since its clinical similarity with other pathologies that can imply a worse prognosis, the authors suggest, in case of suspicion, to refer to a dermatologist or a pediatric surgeon to confirm the diagnosis and follow-up.

\section{Ethical Responsibilities}

Human Beings and animals protection: Disclosure the authors state that the procedures were followed according to the Declaration of Helsinki and the World Medical Association regarding human experimentation developed for the medical community.

Data confidentiality: The authors state that they have followed the protocols of their Center and Local regulations on the publication of patient data.

Rights to privacy and informed consent: The authors have obtained the informed consent of the patients and/or subjects referred to in the article. This document is in the possession of the correspondence author. 


\section{Financial Disclosure}

Authors state that no economic support has been associated with the present study.

\section{Conflicts of Interest}

Authors declare no conflict of interest regarding the present study.

\section{References}

1. Reisenauer A, White KP, Korcheva V, White Jr CR. "Non-infectious

Granulomas" in Dermatology

Third ed. Ed. Bolognia JL, Jorizzo JL, Schaffer JV. Elsevier Saunders. Philadelphia, Pennsylvania, 2012. pp. 1563-64.

2. Grogg KL, Nascimento AG. Subcutaneous granuloma annulare in childhood: clinicopathologic features in 34 cases. Pediatrics. 2001;107(3): E42.

3. Felner EI, Steinberg JB, Weinberg AG. Subcutaneous granuloma annulare: a review of 47 cases. Pediatrics. 1997;100(6):965-7.

4. Misago N, Narisawa Y. Subcutaneous granuloma annulare with overlying localized granuloma annulare. J Dermatol. 2010;37(8):755-7.

5. Rambhia KD, Khopkar US. Asymptomatic Papulo-nodules Localized to One Finger. Indian Journal of Dermatology. 2015;60(5):522.

6. Lynch JM, Barrett TL. Collagenolytic (necrobiotic) granulomas: part 1-the "blue" granulomas. J Cutan Pathol. 2004;31(5):353-61.

7. Sobanko JF, Meijer L, Nigra TP.
Epithelioid Sarcoma: A Review and Update. Jerry T, Sanjay B, Joshua Z, eds. The Journal of Clinical and Aesthetic Dermatology. 2009;2(5):49-54.

8. García-Patos V. Rheumatoid nodule. Semin Cutan Med Surg. 2007;26(2):100-7.

9. Ahmed I, Harshad SR. Subcutaneous sarcoidosis: is it a specific subset of cutaneous sarcoidosis frequently associated with systemic disease? J Am Acad Dermatol. 2006;54(1):55.

10. Paller AS, Pensler JM, Tomita T. Nasal midline masses in infants and children: dermoids, encephaloceles, and gliomas. Arch Dermatol. 1991;127:362-6. 